\title{
Primary Atomization Implementation of Unlike-Doublet Impinging Jets in Numerical Simulation
}

\author{
Ying Feng ${ }^{1}$, Qinghuang Huang ${ }^{2}$, Qiong $\mathrm{Li}^{3}$, Peiyong Wang* \\ ${ }^{1}$ Department of Aerospace Engineering, Xiamen University, Xiamen 361005, China \\ ${ }^{2}$ Xiamen Kaiyun Information Technology Ltd, Xiamen 361027, China \\ ${ }^{3}$ College of Mechanical Engineering and Automation, Huaqiao University, Xiamen 361021, China \\ afengying2842@hotmail.com
}

Keywords: Unlike-doublet impinging jets; Primary atomization; Combustion simulation; Liquid rocket engine.

Abstract. Unlike-doublet impinging jets are frequently used for liquid fuel and oxidizer atomization inside small rocket engines. An accurate setup for the primary atomization of impinging jets is crucial for combustion simulation accuracy. Spray location, spray angle, spray flow rate angular distribution, droplet diameter distribution, deviation angle, and dispersion angle are involved in the implementation of primary atomization setup. Appropriate physical models for these parameters have been identified and implemented in the CFD simulation of a test rocket engine using monomethylhydrazine and nitrogen tetroxide.

\section{Introduction}

Computational fluid dynamics (CFD) has been used in rocket engine combustor simulation to better understand the fluid mechanical and thermodynamic processes inside combustors and to support the design and optimization [1]. The numerical simulation accuracy is dependent on the physical models of the process. Primary atomization model is one of the most important models. Typical atomization models are solid cone and hollow cone which are well defined and implemented in commercial software. However, the impinging jets are used for the atomization of small rocket engines, the atomization is more like the flat-fan type. The flat-fan model is defined in some CFD software such as FLUENT and is not defined in other software such as STAR-CCM+ and STAR-CD. The setup of flat-fan atomization model involves the assignment of spray location, spray angle, spray flow rate angular distribution, droplet diameter distribution, deviation angle, and dispersion angle. The simulation engineers will have to determine the reasonable values for these parameters. The main theory of the impinging jets atomization is reviewed and the instruction of appropriate atomization setup in Star-CCM+ is given here.

\section{Impinging Jets Atomization Theory}

Physical process of the unlike-doublet impinging jets spray. The propellants are ejected from a pair of injectors in a certain angle, a very thin liquid film is formed after impacting at the intersection, as shown in Fig. 1. Liquid film transforms into unstable waves under the influence of air pressure and liquid surface tension. Under the influence of the fastest growing unstable wave, the liquid film breaks into liquid ligament, then into liquid droplets. The whole process is known as the primary atomization. 


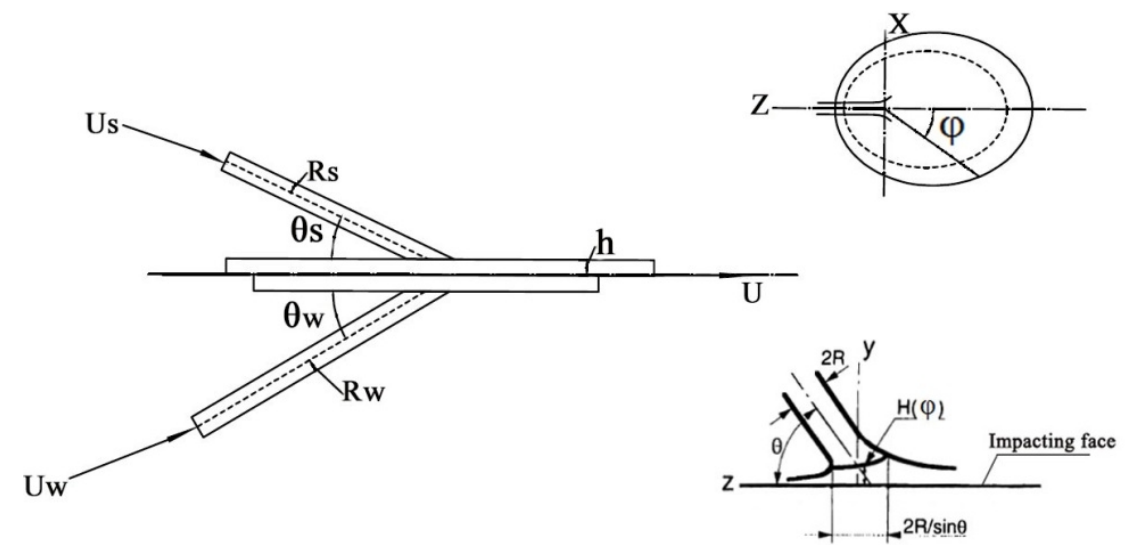

Fig. 1. Schematic diagram of liquid sheet formation by impinging jets.

The models of liquid droplet diameter and distribution, the position of injection, liquid droplet flow rate distribution, deviation angle, and dispersion angle will be reviewed.

Liquid Droplet Diameter And Distribution. The theoretical distribution of the average droplet size $d_{d}$ shed from the symmetric impinging jets (jets with same material, same jet diameter, and same injection velocity) was derived by Lee [2], as follows:

$$
d_{d}=\left(\frac{3 \pi}{\sqrt{2}}\right)^{1 / 3} d_{l}\left[1+\frac{3 \mu}{\left(\rho_{l} \sigma d_{l}\right)^{1 / 2}}\right]^{1 / 6} .
$$

Here, $\mu, \sigma$, and $\rho_{l}$ are the viscosity, the surface tension, and the density of the liquid respectively. And $d_{l}$ is the ligament diameter following:

$$
d_{l}=0.9614\left(\frac{K^{2} \sigma^{2}}{\rho \rho_{l} U}\right)^{1 / 6}\left[1+2.60 \mu\left(\sqrt[3]{\frac{K \rho^{4} U^{7}}{72 \rho_{l}^{2} \sigma^{5}}}\right)\right]^{1 / 5} .
$$

Here, $\rho$ is the density of the gaseous medium, $U$ can be considered nearly equal to the jet velocity, and $K$ is written as,

$$
K=\frac{R^{2} \sin ^{3} \theta}{(1-\cos \varphi \cos \theta)^{2}} .
$$

$R$ represents the radius of the cylindrical liquid jet, $\theta$ is the impinging angle, and $\varphi$ is the azimuthal angle of the impinging sheet. By substituting Eq. (2) and Eq. (3) into Eq. (1), the theoretical average droplet size distribution according to the azimuthal angle can be calculated for symmetric impinging jets.

For the asymmetric jets (jets with different materials, different jet diameters, or different injection velocity) such as the typical hyperbolic MMH and NTO combination, the MMH jet and NTO jet are treated separately in the estimation of the theoretical average droplet size distribution. The theoretical droplet size distribution for each jet in the asymmetric impinging jets can be calculated by applying a symmetric model to each jet, as characterized by $\theta=2 \theta_{w}$ and $\theta=2 \theta_{s}$ for the impinging angle.

The Rosin-Rammler distribution function could be used for the size distribution of the droplets in the impinging jets. The Rosin-Rammler distribution is expressed as:

$$
1-Q=\exp \left[-\left(\frac{D}{d_{d}}\right)^{q}\right]
$$

Here, $Q$ is the volume fraction of the droplets whose diameter is less than $D, q$ is an adjustable constant that functions to improve the fit to experimental data (3.5 is used here [3]).

Liquid Sheet Disintegration. It has been proposed that the liquid properties of the jets are so significant that Weber number alone cannot accurately predict breakup length. James et al. [4] created the correlations for sheet breakup length and ligament breakup length:

$$
\begin{gathered}
X_{b, s} / d_{o}=97.3 W e^{-0.27} \sin ^{-1.20} \theta . \\
X_{b, l} / d_{o}=69.8 O h^{0.094} W e^{-0.14} \sin ^{-0.58} \theta .
\end{gathered}
$$


Here, $d_{o}$ is the injection orifice diameter, $X_{b, s}$ is the sheet breakup length, $X_{b, l}$ is the ligament breakup length, $O h$ is Ohnesorge number $\left(O h=\frac{\mu}{\sqrt{\rho_{l} \sigma d_{o}}}\right)$, We is Weber number $\left(W e=\frac{\rho_{l} U d_{d}}{\sigma}\right)$. For the asymmetric jets, the sheet breakup length and ligament breakup length can be calculated according to their respective parameters. However, high velocity with fully developed breakup [5] (A direct decay into droplets without any ligament formation seems to occur near the impingement point of the two jets when $R e>5000\left(\operatorname{Re}=\frac{\rho_{l} U d_{d}}{\mu}\right)$ and $\left.W e>10000\right)$ will lead to zero sheet breakup length and ligament breakup length.

Liquid Droplet Diameter And Distribution. Lee et al. [6] derived the theoretical distribution of the liquid sheet thickness $h$ for the symmetric impinging jets:

$\beta$ can be determined as follows:

$$
h=\frac{\beta R \sin \theta}{e^{\beta}-1} \exp \left[\beta\left(1-\frac{\varphi}{\pi}\right)\right] .
$$

$$
\left.\cos \theta=\left(\frac{e^{\beta}+1}{e^{\beta}-1}\right) \frac{1}{1+(\pi / \beta)^{2}}\right] .
$$

The differential flow rate can be calculated from:

$$
d Q_{e}=U h_{e} r_{e} d \varphi .
$$

$Q_{e}$ is the volume flow rate, $h_{e}$ is the edge thickness of the sheet, $r_{e}$ is the radius of the sheet. They can be calculated with the radius of the liquid jet and the liquid sheet thickness:

$$
h_{e} r_{e}=R h / \sin \theta \text {. }
$$

This model was developed for symmetric impinging jets. For asymmetric impinging jets, the flow rate can be calculated by applying a symmetric model to each jet, as characterized by $\theta=2 \theta_{w}$ and $\theta=$ $2 \theta_{s}$ for the impinging angle. By substituting Eq. (10) and Eq. (7) into Eq. (9), the theoretical liquid droplet flow rate distribution according to the azimuthal angle can be calculated for asymmetric impinging jets. Theoretically, The flow distribution of $0 \sim 180$ degree and $180 \sim 360$ degree is symmetric, so only the flow distribution of $0 \sim 180$ degree is taken into account.

Deviation Angle and Dispersion Angle. Fig. 2 shows the definition of spraying angle, deviation angle, and dispersion angle. As we all know, some of the droplet will spread on both sides of the impact surface, the entire dispersion range is called the dispersion angle. For an unlike-doublet impinging-jet spray, the resulting spray will deviate from the symmetric axis of the two jets if the two jets are not of the same momentum [7]. The deviation angle $\delta$ (the angle between the angular bisector of the dispersion angle and the horizontal line) can be expressed as:

$$
\delta=\tan ^{-1}\left(\frac{m_{s} U_{s} \sin \theta_{s}-m_{w} U_{w} \sin \theta_{w}}{m_{s} U_{s} \cos \theta_{s}+m_{w} U_{w} \cos \theta_{w}}\right)
$$

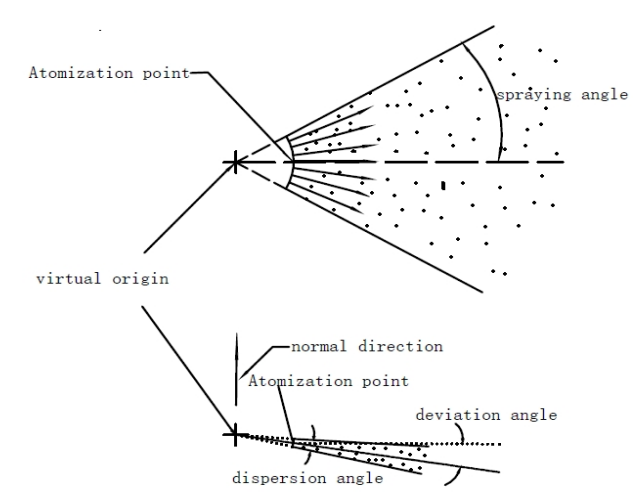

Fig. 2. Spraying angle, deviation angle and dispersion angle.

\section{Numerical Simulation}

Geometry and Mesh. The combustion chamber is axisymmetric as shown in Fig. 3. The combustion flow field is not axisymmetric since the generated primary atomization is not axisymmetric. 
For simplifying simulation, 60 pairs of injectors are assumed to replace the actual 6 pairs of injectors, only a 6 degree piece in azimuthal direction is needed for the simulation.

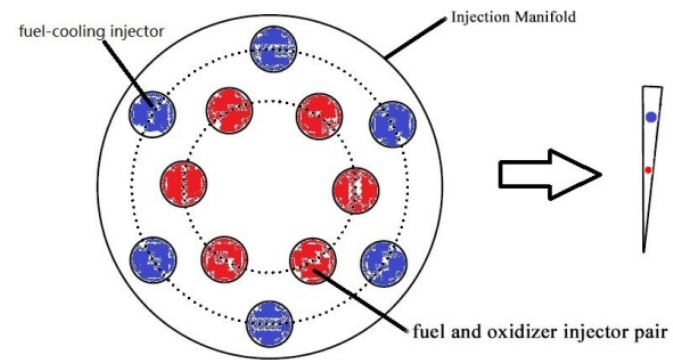

Fig. 3. Location of injectors; left: sketch showing oxidizer and fuel injection areas; right: transformation into an axisymmetric atomization pattern.

The combustion chamber and nozzle is $5.9 \mathrm{~cm}$ long and $3 \mathrm{~cm}$ in diameter near the injection manifold (the figure has not been plotted to scale) as in Fig. 4. Fuel and oxidizer are injected into the engine through a pair of injection orifices. For each pair of orifices, one orifice carries fuel and the other carries oxidizer. The orifices are also slanted toward each other (the impact angle $\theta$ of MMH stream and NTO streams are $30^{\circ}$ and $40^{\circ}$ ) so that the fuel and oxidizer streams within each pair impinge immediately.

The computational grid used throughout the subsequent investigations is depicted in Fig.4. 263 grid points in flow direction and 32 grid points in radial direction (11219 cells in total). The minimal mesh spacing at the cylinder wall is around $8 \mu \mathrm{m} \sim 10 \mu \mathrm{m}$. The simulation reveals that the local $\mathrm{y}+$ values between 0.3 and 37 .

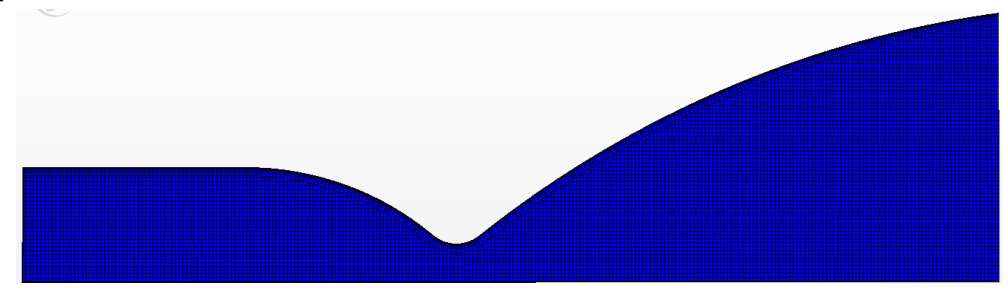

Fig.4. Computation mesh.

The Star-CCM+ software does not offer a good primary atomization model for implementation of the impinging jets atomization such as the flat-fan model. The primary atomization has to be carefully setup by a dozen of point injectors. To save the computation time, each MMH and NTO injectors in the same direction is located at the same position (as a pair). According to 2.1.3, it is shown that the flow rate of $0 \sim 30$ degree is almost $100 \%$ of the total flow rate (NTO is $99.22 \%$ and MMH is $96.72 \%$ ), so the flow distribution of $0 \sim 30$ degree (which is called spraying angle) for the numerical simulation is chosen to simplify the model. According to Eq. (12), the deviation angle is 0.183 radian approximately in this study. The choice of dispersion angle is based on past modeling experience [3], which is 6 degree.

Placing a pair of point injectors at every 5 degree in the direction of atomization, their locations are shown in Fig. 5 by pink points (deviation angle can't be seen clearly). The orifices are not explicitly represented in the simulation, because fuel/oxidizer are injected into the grid cell adjacent to the chamber wall at these locations. By Eq. (5) and Eq. (6), the NTO sheet breakup length is $9.87 \mathrm{~mm}$ and the NTO ligament breakup length is $8.69 \mathrm{~mm}$, but the $R e$ and $W e$ of NTO and MMH jets are both located near the fully developed breakup regime (Re-NTO is 23500 , We-NTO is 5028, Re-MMH is 10300 , We-MMH is 7306), a total breakup length of $5 \mathrm{~mm}$ is used for both jets.

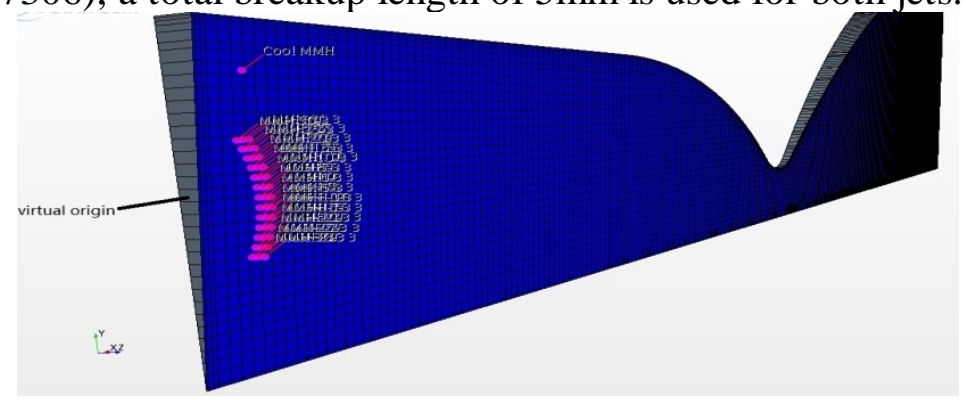

Fig. 5 Location of point injectors. 
The impinging pairs locate at the radius $15 \mathrm{~mm}$ on the side wall, and the impact angle $\theta$ of $\mathrm{MMH}$ stream and NTO streams are $30^{\circ}$ and $40^{\circ}$ respectively. The cooling MMH injector is located at the radius $24 \mathrm{~mm}$ on the side wall. The corresponding MMH mass flow rate for the 6 degree geometry is $0.2477 \mathrm{~g} / \mathrm{s}$. The NTO flow rate is $0.4087 \mathrm{~g} / \mathrm{s}$. The cooling MMH flow rate is $0.0762 \mathrm{~g} / \mathrm{s}$. Calculated with Eq. (1) and Eq. (9), the spatial distribution of mass flow rate and droplet diameter are shown in Table 1.

Table 1 the spatial distribution of mass flow rate and droplet diameter

\begin{tabular}{|c|c|c|c|c|c|c|}
\hline $\begin{array}{c}\text { spra } \\
\text { y } \\
\text { ang } \\
\text { le/ } \\
0\end{array}$ & $\begin{array}{c}\mathrm{MMH}^{*} 1 \\
0^{7} / \\
\mathrm{kg} / \mathrm{s}\end{array}$ & $\begin{array}{c} \pm 3^{\circ} \\
\mathrm{MMH} \\
\text { dispersio } \\
\mathrm{n} \\
\underset{7}{\operatorname{angle}}{ }^{*} 10 \\
/ \mathrm{kg} / \mathrm{s}\end{array}$ & $\begin{array}{c}\text { MMH } \\
\text { average } \\
\text { droplet } \\
\text { diameter*1 } \\
04 \\
\text { M }\end{array}$ & $\begin{array}{c}\mathrm{NTO}_{7 /}^{*} 10 \\
\mathrm{~kg} / \mathrm{s}\end{array}$ & $\begin{array}{c} \pm 3^{\circ} \\
\text { NTO } \\
\text { dispersio } \\
\mathrm{n} \\
\text { angle } \\
7 / \\
\mathrm{kg} / \mathrm{s}\end{array}$ & $\begin{array}{c}\text { NTO } \\
\text { droplet } \\
\text { diameter*1 } \\
0^{4} / \\
\mathrm{m}\end{array}$ \\
\hline 0 & 951.072 & 52.8373 & 1.07 & 1841.58 & 102.31 & 2.11 \\
\hline \pm 5 & 273.218 & 15.1788 & 1.046 & 459.479 & 25.5416 & 2.02 \\
\hline \pm 10 & 162.15 & 9.0083 & 1.007 & 229.551 & 12.7529 & 1.88 \\
\hline \pm 15 & 99.5105 & 5.52836 & 0.958 & 114.174 & 6.36747 & 1.73 \\
\hline \pm 20 & 61.0692 & 3.39273 & 0.904 & 70.7266 & 3.92926 & 1.57 \\
\hline \pm 25 & 34.4779 & 2.08211 & 0.848 & 37.5731 & 2.08739 & 1.42 \\
\hline \pm 30 & 11.0773 & 0.6154 & 0.792 & 11.9858 & 0.6659 & 1.28 \\
\hline
\end{tabular}

The injection velocity of streams is used as the droplet velocity [8]. The velocity of all MMH droplets is $28.29 \mathrm{~m} / \mathrm{s}$, the velocity of all NTO droplets is $13.4 \mathrm{~m} / \mathrm{s}$ and the velocity of cooling MMH droplets is $19.27 \mathrm{~m} / \mathrm{s}$. The temperature of all liquid droplets is $280 \mathrm{~K}$.

EBU (Eddy Break-Up) model is used for none-premixed combustion (the reaction adopted is: $4 \mathrm{CH} 3 \mathrm{NHNH} 2+5 \mathrm{~N} 2 \mathrm{O} 4=9 \mathrm{~N} 2+4 \mathrm{CO} 2+12 \mathrm{H} 2 \mathrm{O}$, the model parameter $\mathrm{A}$ is 4 ), K-Epsilon turbulence model is used for turbulence modeling, Lagrangian multiphase model is used, KHRT model is used for droplet break up Model.

\section{Result and Discussion}

Fig. 6 shows the predicted temperature and pressure contours within the thruster. The cold injection wall region is distinctly visible. In the lower left corner, there is a region with high temperature, this is because the diameter of the propellant droplets is so large that these droplets evaporate into a large amount of gaseous MMH and NTO, stranding in the lower left corner, then a large amount of heat is generated by gas reaction to produce local high temperature. The calculated chamber pressure is 0.845 $\mathrm{MPa}$. The pressure distribution is a direct result of the complete chamber flow field calculation and hence evaluates the atomization model and combustion model.

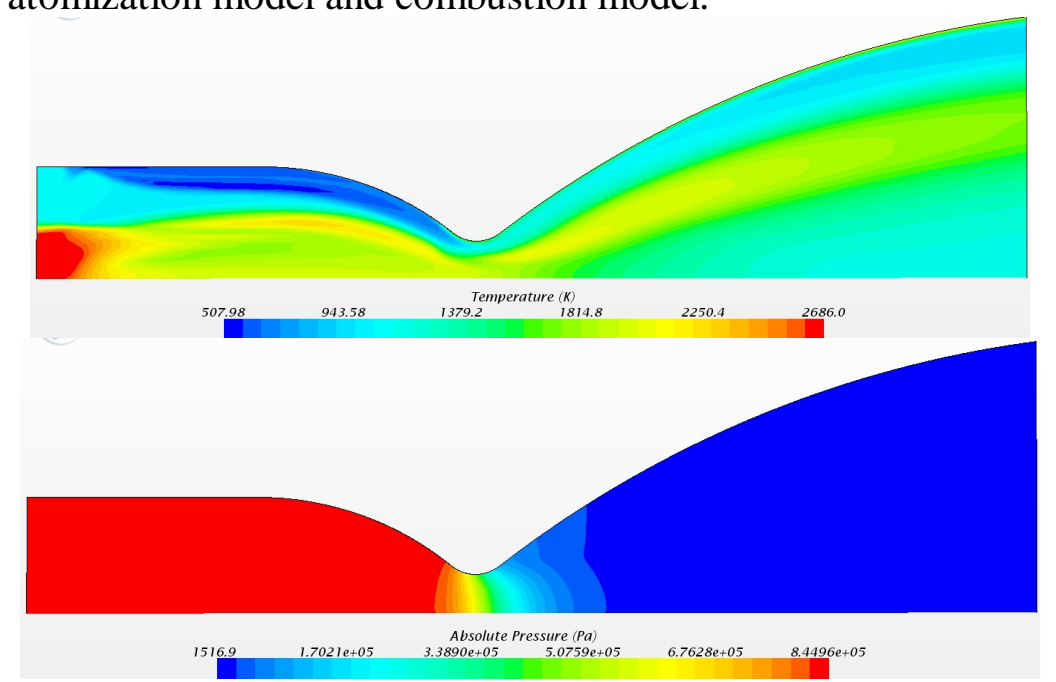

Fig. 6. The predicted temperature (up) and pressure (down) contours within the thruster.

Fig. 7shows the predicted droplet trajectory and droplet diameter distribution after primary atomization. It can be seen that the assumed spray model is consistent with the actual spray in most 
respects, in addition to wall conditions since the liquid film is not considered here, all of the liquid droplets are treated as rebounding when impacting walls, which is not the actual situation. Even so, the spray model is an effective model.

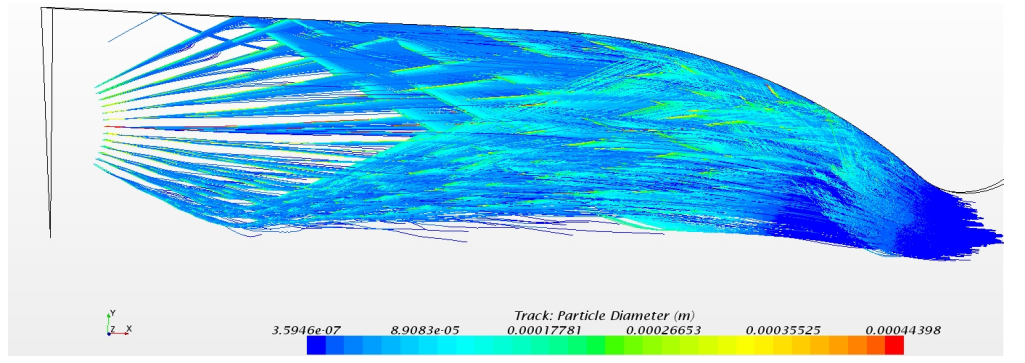

Fig. 7Predicted droplet trajectory and diameter distribution.

\section{Conclusions}

The primary atomization theory of the impinging jets has been reviewed and the parameters to define the atomization have been identified: Spray location, spray angle, spray flow rate angular distribution, droplet diameter distribution, deviation angle, and dispersion angle. The detailed calculation method for these parameters is determined. The primary atomization model has been applied in the simulation of the test rocket engine. It is found that the model predicts a reasonable droplet distribution and the simulation result is conducive to future research.

\section{Acknowledgments}

This work is sponsored by the National Natural Science Foundation of China Grant 51106131 and 51206057.

\section{References}

[1] O. Knab, D. Preclik and D. Estublier, Flow field prediction within liquid film cooled combustion chambers of storable bi-propellant rocket engines, AIAA-98-3370.

[2] C.H. Lee, An experimental study on the distribution of the drop size and velocity in asymmetric impinging jet sprays, Journal of Mechanical Science and Technology, 22, 608 (2008).

[3] D.P. Schmidt, M.L. Corradini, and C. J. Rutland, A two-dimensional, non-equilibrium model of flashing nozzle flow. In 3rd ASME/JSME Joint Fluids Engineering Conference. 1999.

[4] M.D. James, T.D. Kubal, S. Son, W. Anderson, and T. Pourpoint, Calibration of an impinging jet injector suitable for liquid and gelled hypergolic propellants, AIAA 2009-4882.

[5] G. Bailardi, M. Negri, and H.K. Ciezki, Several aspects of the atomization behavior of various newtonian fluids with a like-on-like impinging jet injector, $23 \mathrm{rd}$ annual conference on Liquid Atomization and Spray Systems, Brno, Czech Republic September 2010.

[6] C.H. Lee, Y.H. Jung, and S.H. Chung, An experiment on flow distribution and mixing in jet sprays, Atomization and Sprays, 9193 (1999).

[7] W.H. Lai, W. Huang, and C.J. Chu, Atomization and mixing characteristics of an unlike-doublet impinging-jet spray, International Journal of Turbo and Jet Engines, 21, 127 (2004).

[8] Y.J. Choo and B. Kang, A study on the velocity characteristics of the liquid elements produced by two impinging jets, Experiments in Fluids, 34, 655 (2003). 